\title{
EFFECT OF FEMORAL OFFSET ON RANGE OF MOTION AND ABDUCTOR MUSCLE STRENGTH AFTER TOTAL HIP ARTHROPLASTY
}

\author{
BRIAN J. MCGRORY, BERNARD F. MORREY, THOMAS D. CAHALAN, KAI-NAN AN, MIGUEL E. CABANELA
}

From the Mayo Clinic and Mayo Foundation, Rochester, USA

\begin{abstract}
At a minimum of one year after operation, we studied 64 patients with 86 total hip arthroplasties (THA) by standard anteroposterior hip and pelvic radiographs and measurement of range of motion and of isometric abduction strength.

The femoral offset correlated positively with the range of abduction $(p=0.046)$. Abduction strength correlated positively with both femoral offset $(p=0.0001)$ and the length of the abductor lever arm $(p=0.005)$. Using multiple regression, abduction strength correlated with height $(p=0.017)$, gender $(p=0.0005)$, range of flexion $(p=0.047)$ and the abductor lever arm $(p=0.060)$.

Our findings suggest that greater femoral offset after THA allows both an increased range of abduction and greater abductor strength.
\end{abstract}

J Bone Joint Surg [Br] 1995;77-B:865-9.

Received 22 June 1994; ; Accepted after revision 23 March 1995

There has been recent interest in femoral offset as a factor in total hip arthroplasty (THA) (Yanagimoto 1991; Abraham and Dimon 1992; Steinberg and Harris 1992; Davey et al 1993; Rothman et al 1993; Wong et al 1993). Femoral offset is defined as the perpendicular distance between the long axis of the femur and the centre of rotation of the femoral head. Charnley (1979) considered it to be a factor under the control of the surgeon at the time of hip replacement surgery; the more lateral position of the femur with greater offset was said to increase the range of motion and

B. J. McGrory, MD, Senior Resident

Orthopaedic Associates of Portland, PO Box 1260, 33 Sewall Street, Portland, Maine 04104-1260, USA.

B. F. Morrey, MD, Chairman, Department of Orthopaedics

T. D. Cahalan, RPT, Physical Therapist

K.-N. An, PhD, Director, Biomechanics Laboratory

M. E. Cabanela, MD, Professor of Orthopaedics

Mayo Clinic and Mayo Foundation, 200 First Street SW, Rochester, Minnesota 35905, USA.

Correspondence should be sent to Dr B. F. Morrey.

C1995 British Editorial Society of Bone and Joint Surgery 0301-620X/95/61066\$2.00 decrease the incidence of impingement of the femur on the pelvis. An increase in femoral offset (and consequently of the lever arm of the abductor muscles) will also, theoretically, increase the mechanical advantage and strength of the abductors. Finally, a greater femoral offset will increase stability by preventing impingement and improving softtissue tension.

Femoral offset has been shown to correlate with hip stability (Fackler and Poss 1980; Huk et al 1993), but we know of no clinical study which has related the range of motion or abduction strength to femoral offset after THA. We therefore reviewed 64 consecutive patients at a minimum of one year after surgery in an attempt to answer these questions.

\section{PATIENTS AND METHODS}

We reviewed 64 patients who had a total of 86 THAs by one of two surgeons (BFM, MEC) at a minimum of one year (mean one year nine months, range one year to three years two months) after surgery, by radiography, examination, and standard abductor strength testing. The number of joint replacements included was based on a power analysis calculation (see statistical section). The minimum followup was chosen because results for THA have been shown to stabilise at one year (Insall et al 1983; Schurman, Parker and Ornstein 1985; Parsley, Engh and Dwyer 1992). We excluded patients with a diagnosis of rheumatoid arthritis to avoid confounding factors in strength measurements. Patients who would have had to travel more than 500 miles were also excluded; the one-year assessment for such patients is often carried out locally.

The patients had been operated on over a two-year period, from 1988 to 1989, during which 263 THAs (excluding patients with a diagnosis of rheumatoid arthritis) had been performed by the two surgeons. Informed consent was obtained and the project and consent process were approved by the IRB of the Mayo Clinic.

There were 36 women and 28 men and the median age was 60 years (22 to 87). Their average weight and height were $77.7 \mathrm{~kg}$ (44.5 to 109.1) and $168 \mathrm{~cm}$ (140 to 190). All were seen in follow-up from April 1, 1989 to April 1, 1993 and no evaluations were excluded. The right hip had been replaced in $48(55.8 \%)$ and the left in 38 . The diagnosis was osteoarthritis in 57 (66.3\%), post-traumatic deformity 
in nine (10.5\%), developmental dysplasia in nine (10.5\%), avascular necrosis in five (5.8\%), Paget's disease in two (2.3\%), arthritis following slipped capital femoral epiphysis in two, and systemic lupus erythematosus in two. There were 70 primary and 16 revision operations. Eighteen (20.9\%) femoral components were cemented and 68 uncemented. An anterolateral approach was used in 32 (37.2\%) and a posterior approach in 54; trochanteric osteotomy was not used. A variety of femoral prostheses was employed. There were 34 (39.5\%) Osteonics Omnifit (Osteonics Corp, Allendale, New Jersey), 14 (16.3\%) Osteonics Omniflex, 13 (15.1\%) Zimmer Mayo-Morrey (Zimmer Inc, Warsaw, Indiana), 7 (8.2\%) Zimmer Harris precoat, 5 (5.8\%) Zimmer Harris-Galante, 5 (5.8\%) Zimmer Bias, and eight other prostheses $(9.3 \%)$ of varying design chosen by the surgeon.

Radiological assessment. Anteroposterior pelvic and hip radiographs were taken on the day of examination using a $100 \mathrm{~mm}$ magnification marker with the ankles $20 \mathrm{~cm}$ apart and the feet $15^{\circ}$ internally rotated. The femoral offset and the abductor lever arm were measured by a single observer (BJM) from each radiograph (Fig. 1). In addition, the distance from the centre of rotation of the femoral head to

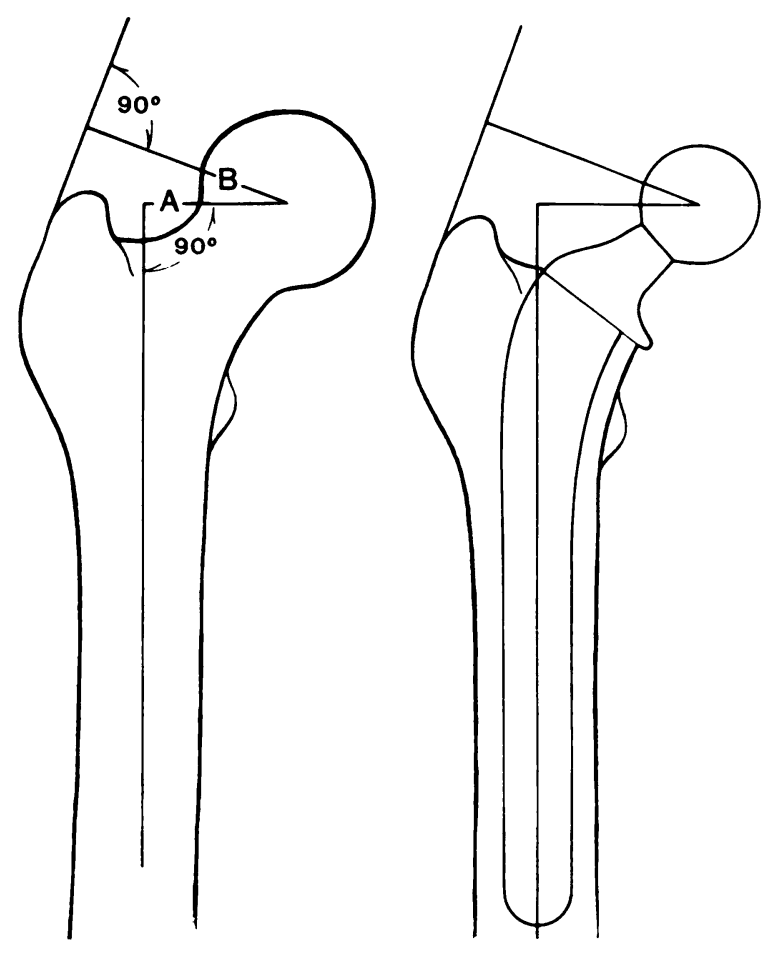

Fig. 1

Diagram showing measurements of femoral offset (A) and the abductor lever arm (B). Femoral offset is the perpendicular distance from the centre line of the femur to the centre of rotation of the femoral head. Abductor lever arm is the perpendicular distance from a line tangential to the greater trochanter to the centre of rotation of the femoral head. The tangential line corresponds to the abductor shadow on the radiograph (from Steinberg and Harris 1992, with permission). a vertical line through the symphysis pubis was recorded as the body-weight lever arm. These measurements were then corrected for magnification.

Functional assessment. A complete examination included the recording of the patients' height, weight, and maximum thigh circumference on the affected side.

Motion. The ranges of motion in flexion, abduction, adduction, internal rotation, and external rotation were measured using a goniometer by a single observer (TDC).

Strength. Three measurements of isometric abduction strength at $0^{\circ}$ (neutral) were made for each THA by the method previously described (Cahalan et al 1989). A Cybex II isokinetic dynamometer (Lumex, Ronkonkoma, New York) was modified to allow adjustment of the loading level arm to provide a comfortable position for the patient and maximal stability to produce optimal strength. A body stabilisation frame was developed and used to allow subjects to stand with support when testing abduction strength. The Cybex machine was calibrated at weekly intervals. We used the numerical average of three measurements as the abductor strength for data analysis.

Statistical analysis. A power analysis showed that a study of 84 THAs should provide at least an $80 \%$ chance (statistical power) of detecting any correlation between offset and range of motion or strength, accepting an $r$ value of 0.30 . Linear regression beta coefficients and corresponding probability using StatView software (Abacus Concepts Inc, Berkeley, California) were determined for femoral offset versus range of motion, and for abduction strength versus gender, age, diagnosis, revision, surgical approach, component fixation, height, weight, thigh circumference, offset, abduction lever arm, time from surgery, and body-weight lever arm. We then used multiple regression to determine which of the variables found to have significant interrelationships were the most important.

\section{RESULTS}

Radiological assessment. The corrected femoral offset averaged $3.9 \mathrm{~cm} \mathrm{(2.3} \mathrm{to} \mathrm{5.5),} \mathrm{the} \mathrm{abductor} \mathrm{lever} \mathrm{arm} \mathrm{aver-}$ aged $4.8 \mathrm{~cm} \mathrm{(3.4} \mathrm{to} 7.1)$ and the body-weight lever arm averaged $9.2 \mathrm{~cm}$ (7.8 to 10.7$)$.

Femoral offset correlated positively with the length of the abductor lever arm $(p=0.0001 ; r=0.43 ;$ Fig. 2$)$. The body-weight lever arm did not correlate with either offset or the abduction lever arm.

Functional assessment

Motion. The average range of motion with range and standard deviation is shown in Table I. Simple regression analysis showed that femoral offset was significantly and positively related to range of abduction $(p=0.046$; $r=0.22$; Fig. 3). We could demonstrate no significant correlation between femoral offset and hip flexion, adduction, or internal or external rotation.

Strength. There was a highly significant positive correlation between femoral offset (and consequently abductor lever 


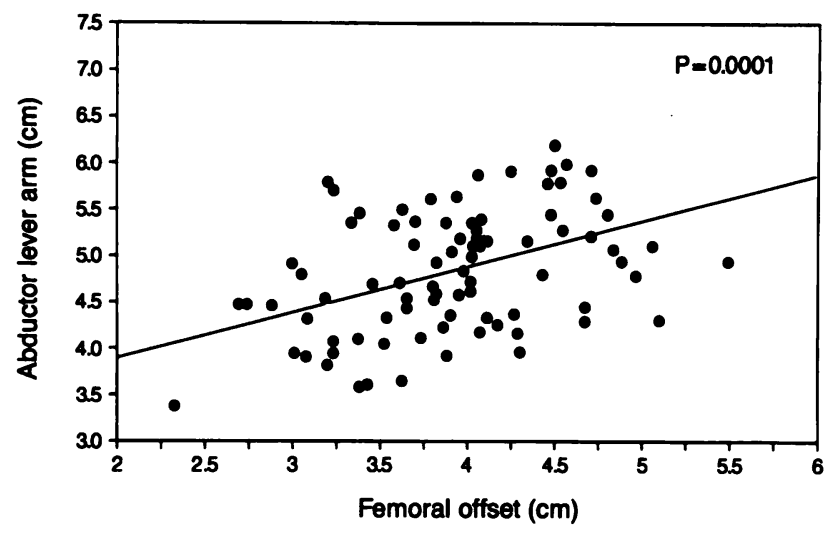

Fig. 2

Femoral offset related to length of abductor lever arm.

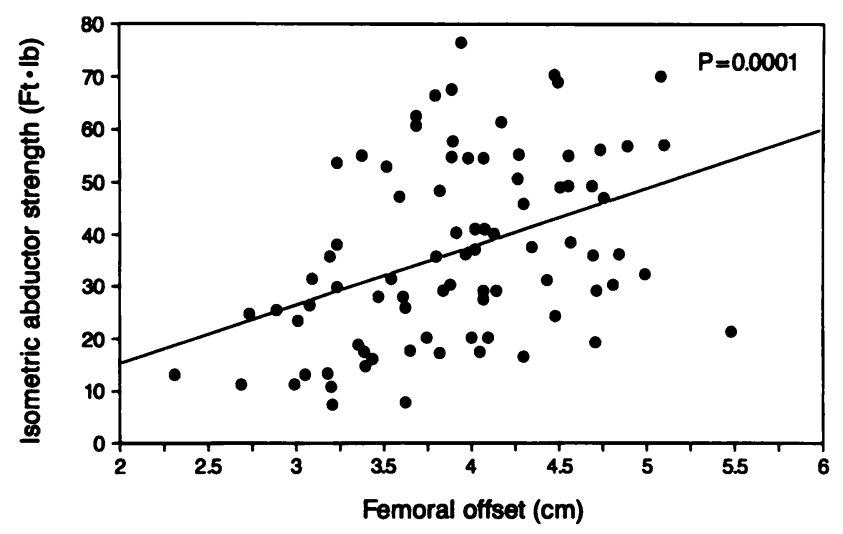

Fig. 4

Femoral offset related to isometric abductor strength.

Table I. Range of hip motion after THA in degrees

\begin{tabular}{llll}
\hline & Average & Range & $\begin{array}{l}\text { Standard } \\
\text { deviation }\end{array}$ \\
\hline Flexion & 99.8 & 48 to 121 & 15.3 \\
Abduction & 25.7 & 10 to 45 & 7.7 \\
Adduction & 19.8 & 8 to 35 & 5.7 \\
Internal rotation & 26.5 & 7 to 51 & 9.2 \\
External rotation & 24.9 & 6 to 48 & 8.1 \\
\hline
\end{tabular}

Table II. Factors which correlated with strength of isometric abduction after THA by simple regression

\begin{tabular}{lll}
\hline & p value & r value \\
\hline Patient height & 0.0001 & 0.72 \\
Patient gender (male > female) & 0.0001 & 0.71 \\
Femoral offset & 0.0001 & 0.40 \\
Abduction ROM $*$ & 0.0009 & 0.36 \\
Patient weight & 0.001 & 0.35 \\
Flexion ROM* & 0.004 & 0.32 \\
Abductor lever arm & 0.005 & 0.30 \\
Patient diagnosis $(\mathrm{OA} \dagger>$ others) & 0.03 & 0.24 \\
\hline
\end{tabular}

* range of motion

tosteoarthritis

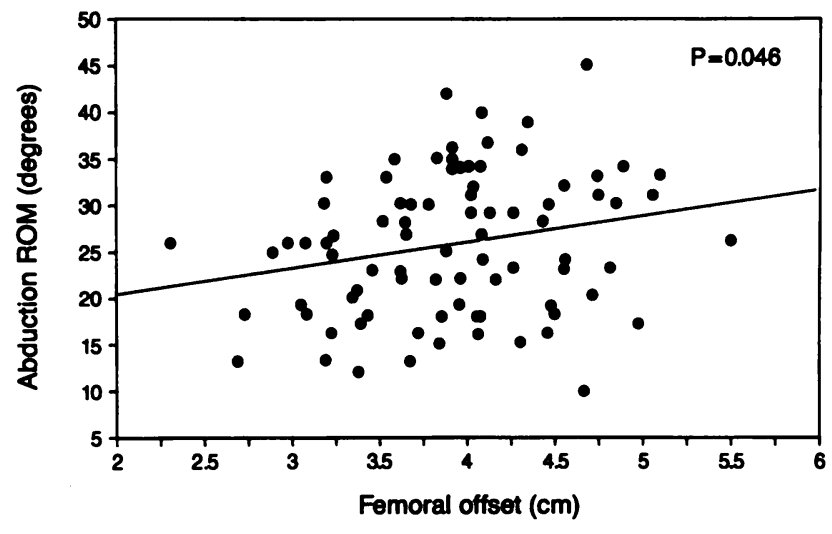

Fig. 3

Femoral offset related to range of abduction.

arm) and abductor strength ( $p=0.0001 ; r=0.40$; Fig. 4). The other factors which correlated with abductor strength are shown in Table II.

Additional variables and multivariate analysis. Abductor strength was not significantly related to side of arthroplasty, thigh circumference, use of cement for the femoral component, revision surgery, age, surgical approach, bodyweight lever arm, and time from surgery. Multiple regression was used to determine the relative importance of each of the significant variables because a number of them were inter-related. The statistical data for each variable are presented in Table III. Of the four, only the length of the abductor lever arm is under the control of the surgeon.

\section{DISCUSSION}

The advantages of increasing femoral offset at THA are reported to include an increased range of motion, better mechanical advantage for the abductors and decreased instability because of better soft-tissue tension. Our study is the first, to our knowledge, to address the first two issues in a clinical setting.

Table III. Factors which correlated with strength of isometric abduction after THA by multiple regression analysis

\begin{tabular}{llll}
\hline & $\begin{array}{l}\text { Standardised } \\
\text { coefficient }\end{array}$ & p value & r value \\
\hline Patient height & 0.47 & 0.017 & 0.95 \\
Patient gender (male > female) & 0.44 & 0.0005 & 0.86 \\
Femoral offset & 0.030 & 0.74 & 0.67 \\
Abduction ROM* & 0.16 & 0.098 & 0.78 \\
Patient weight & 0.19 & 0.13 & 0.87 \\
Flexion ROM* & 0.18 & 0.047 & 0.74 \\
Abductor lever arm & 0.16 & 0.060 & 0.68 \\
Patient diagnosis (OA $\dagger>$ others) & 0.012 & 0.89 & 0.68 \\
\hline
\end{tabular}

* range of motion

†osteoarthritis 
The relationship between increasing femoral offset and stability after hip arthroplasty has been shown (Fackler and Poss 1980; Huk et al 1993). In our series there was a single postoperative dislocation $(1.1 \%)$ in a patient with an absolute offset of $4.1 \mathrm{~cm}$ (series average $3.9 \mathrm{~cm}$ ). We can therefore make no definitive statement on hip stability.

The lateral position of a hip with greater offset has been said to allow an increase in motion (Charnley 1979; Kelikian et al 1983) and we found that range of abduction was significantly greater in patients with greater femoral offset $(p=0.046)$. We could not, however, show any correlation with other planes of motion.

Charnley considered that the most effective method available to the surgeon to improve the abductor lever arm, and therefore the biomechanics, was to increase the offset. This should decrease the abductor force required for walking, and therefore decrease the energy requirement for gait as well as the overall joint reactive force.

Other authors have inferred that this relationship is true from apparent abductor weakness and lurch evident in patients with poor offset (Tauber et al 1980; Kelikian et al 1983; Rothman et al 1993). We identified a variety of factors that correlated with abductor strength after THA, among which were femoral offset $(p=0.0001)$ and the length of the abductor lever arm $(p=0.005)$. Multiple regression showed that the four most important factors in abductor strength after THA were height, gender, range of flexion, and abductor lever arm (Table III). All the other factors found to be significant by simple regression analysis were related to these four.

The advantages of increased stability, range of motion, and abduction strength are conferred by an increased femoral offset, but a possible disadvantage is an increase in the out-of-plane bending moment in the prosthesis. This effect in the stem is generally not important in modern THAs because of the increased fatigue resistance of currently used metals (Steinberg and Harris 1992). An increase in offset, however, could cause increased strain in the medial proximal femur, and more particularly in the medial proximal bone cement in cemented cases.

These potential concerns have been allayed by two recent scientific reports. Davey et al (1993) loaded cadaver femora containing cemented femoral components in simulated gait. Direct measurements of strain were made using strain gauges on the bone and the metal as well as in the cement mantle. It was possible to quantify the effect of changing offset on both abductor force and the resultant force, as well as on the strains in the cement, the bone and the prosthesis. Increased femoral offset gave substantial reductions in both abductor and resultant forces, and the strain in the proximal medial cement mantle was not significantly increased. Wong et al (1993) used finiteelement analysis and a canine uncemented THA model to demonstrate that both the abductor force and hip reaction force were significantly reduced with an increase in offset. Although stress was slightly higher at the distal portion of the stem it did not produce an excessive increase in bone strains. In addition, the amount of bone ingrowth was not affected by a change in femoral offset. Both reports indicate that the adverse effect of an increasing bending moment was neutralised by a reduction in hip force.

Despite these experimental results, the clinical concern of increasing femoral component loosening has been expressed by Rothman et al (1993). They showed a 6\% rate of loosening in femora with implant offset of $38 \mathrm{~mm}$ or more, against a $2 \%$ rate in those with less offset, in 146 patients followed for two to six years. These results must be interpreted cautiously; there were only five loose stems overall, there were significantly more males in the highoffset group, and the actual femoral offset was not reported, only the prosthesis offset. This is obviously important in that varus or valgus prosthesis positioning affects actual femoral offset.

Our data allow us to compare the abduction strength after THA with that for normal volunteers, previously reported (Cahalan et al 1989). We demonstrated the significant relationship of gender to strength, but could not identify the age-dependent relationship evident in normal volunteers. We believe that this was because all but one patient in our study would be in the 'older group' of the series by Cahalan et al (1989) (over 40 years of age). Of the anthropomorphic measurements reported in both studies, height had the greatest correlation. When gender-specific abduction strength is compared between normal volunteers and THA patients the latter have lower actual strengths by an average of $24.6 \%$ for men and $37.8 \%$ for women. This may be due to actual age difference, the effect of underlying diagnoses, the effect of surgery, or other factors.

Conclusions. We have shown that femoral offset correlated positively with increase in range of abduction and that objective strength of abduction correlated positively with both femoral offset and the length of the abductor lever arm.

We would like to thank Amy Weaver, MS, Department of Biostatistics, for her advice and help with the statistical analysis of data.

No benefits in any form have been received or will be received from a commercial party related directly or indirectly to the subject of this article.

\section{REFERENCES}

Abraham WD, Dimon JH III. Leg length discrepancy in total hip arthroplasty. Orthop Clin North Am 1992;23:201-9.

Cahalan TD, Johnson ME, Liu S, Chao EY. Quantitative measurements of hip strength in different age groups. Clin Orthop 1989;246: 136-45.

Charnley J. Low friction principle. In: Charnley J. Low friction arthroplasty of the hip. New York: Springer-Verlag, 1979:3-15.

Davey JR, O'Connor DO, Burke DW, Harris WH. Femoral component offset: its effect on strain in bone-cement. J Arthroplasty 1993; 8:23-6.

Fackler CD, Poss R. Dislocation in total hip arthroplasties. Clin Orthop 1980;151:169-78.

Huk OL, Braun JT, Lieberman JR, Salvati EA, Ghelman B. Comparison of acetabular and femoral component alignment in dislocators versus nondislocators with total hip arthroplasties. Procs Canadian Orthopaedic Society, 48th Annual Meeting, Montreal, Quebec, June 1993. 
Insall JN, Hood RW, Flawn LB, Sullivan DJ. The total condylar knee prosthesis in gonarthrosis: a five to nine-year follow-up of the first one hundred consecutive replacements. J Bone Joint Surg [Am] 1983;65-A: 619-28.

Kelikian AS, Tachdjian MO, Askew MJ, Jasty M. Greater trochanteric advancement of the proximal femur: a clinical and biomechanical study. In: Hungerford DS, ed. The Hip. Procs of the eleventh open scientific meeting of the Hip Society. St Louis: CV Mosby, 1983:77-105.

Parsley BS, Engh GA, Dwyer KA. Preoperative flexion: does it influence postoperative flexion after posterior-cruciate-retaining total knee arthroplasty? Clin Orthop 1992;275:204-10.

Rothman RH, Hearn SL, Eng KO, Hozack WJ. The effect of varying femoral offset on component fixation in cemented total hip arthroplasty. Poster: American Academy of Orthopaedic Surgery 60th Annual Meeting, San Francisco, CA, February 1993.
Schurman DJ, Parker JN, Ornstein D. Total condylar knee replacement: a study of factors influencing range of motion as late as two years after arthroplasty. J Bone Joint Surg [Am] 1985;67-A:1006-14.

Steinberg B, Harris WH. The 'offset' problem in total hip arthroplasty. Contemp Orthop 1992;24:556-62.

Tauber C, Ganel A, Horoszowski H, Farine I. Distal transfer of the greater trochanter in coxa vara. Acta Orthop Scand 1980;51:661-6.

Wong PKC, Otsuka NY, Davey JR, Fornasier VL, Binnington AG. The effect of femoral component offset in uncemented total hip arthroplasty. Procs Canadian Orthopaedic Society 48th Annual Meeting, Montreal, Quebec, June 1993.

Yanagimoto S. Basic study of cementless hip prosthesis design: analysis of the proximal femur in Japanese patients with osteoarthritis of the hip. Nippon Seikeigeka Gakkai Zasshi 1991;65:731-44. 